\title{
BEEM-Static: Accurate inference of ecological interactions from cross-sectional metagenomic data
}

Chenhao Li ${ }^{1, *}$, Tamar V. Av-Shalom ${ }^{1,2}$, Jun Wei Gerald Tan ${ }^{1}$, Junmei Samantha $\mathrm{Kwah}^{1}$, Kern Rei Chng ${ }^{1}$ and Niranjan Nagarajan ${ }^{1, *}$

${ }^{1}$ Genome Institute of Singapore, Laboratory of Metagenomic Technologies and Microbial Systems, Singapore, 138672, Singapore,

${ }^{2}$ University of Toronto, Department of Cell \& Systems Biology, Toronto, ON M5S 3G5, Canada.

Chenhao Li: lich@gis.a-star.edu.sg

Tamar V. Av-Shalom: tamar.avshalom@mail.utoronto.ca

Jun Wei Gerald Tan: tanjunweigerald1997@gmail.com

Junmei Samantha Kwah: $\underline{\text { Samantha Kwah@gis.a-star.edu.sg }}$

Kern Rei Chng: CHNG_Kern_Rei@gis.a-star.edu.sg

Niranjan Nagarajan: nagarajann@ @is.a-star.edu.sg

*Corresponding Author: Chenhao Li, Niranjan Nagarajan 


\begin{abstract}
Motivation: The structure and function of diverse microbial communities is underpinned by ecological interactions that remain uncharacterized. With rapid adoption of metagenomic sequencing for studying microbiomes, data-driven inference of microbial interactions based on abundance correlations is widely used, but with the drawback that ecological interpretations may not be possible. Leveraging cross-sectional metagenomic datasets for unravelling ecological structure in a scalable manner thus remains an open problem.

Methods: We present an expectation-maximization algorithm (BEEM-Static) that can be applied to cross-sectional datasets to infer interaction networks based on an ecological model (generalized Lotka-Volterra). The method exhibits robustness to violations in model assumptions by using statistical filters to identify and remove corresponding samples.

Results: Benchmarking against 10 state-of-the-art correlation based methods showed that BEEM-Static can infer presence and directionality of ecological interactions even with relative abundance data (AUC-ROC>0.85), a task that other methods struggle with (AUCROC $<0.63$ ). In addition, BEEM-Static can tolerate a high fraction of samples (up to $40 \%$ ) being not at steady state or coming from an alternate model. Applying BEEM-Static to a large public dataset of human gut microbiomes $(n=4,617)$ identified multiple stable equilibria that better reflect ecological enterotypes with distinct carrying capacities and interactions for key species.
\end{abstract}

Conclusion: BEEM-Static provides new opportunities for mining ecologically interpretable interactions and systems insights from the growing corpus of metagenomic data. 


\section{Introduction}

Microbial communities represent complex systems that impact various aspects related to human health, e.g. agriculture (Mohanram and Kumar, 2019), food processing (De Filippis et al., 2018), disease biology (Chng et al., 2016; Clemente et al., 2012) and healthcare (Chng et al., 2020). Interactions between members of a microbial community determine emergent phenomena such as homeostasis in the ecosystem (Gould et al., 2018; Cordero and Datta, 2016) and overall function of the microbiome (Fraune et al., 2015). Correspondingly, ecological modeling of microbiomes is a key step towards understanding community function (Buffie et al., 2014; Röttjers and Faust, 2018), forecasting dynamics (Xiao et al., 2020; Angulo et al., 2019) and rationally designing interventions that alter community structure and function (Stein et al., 2018).

Advances in high-throughput sequencing and metagenomics have enabled several datadriven approaches to infer microbial interactions, bypassing limitations of experimental approaches in terms of time, resources and cultivability (Faust and Raes, 2012; Li et al., 2016). In particular, correlation-based methods are widely used for their convenient applicability to cross-sectional datasets (Berry and Widder, 2014; Faust and Raes, 2012), despite their inability to capture directionality of ecological interactions such as predation and parasitism (Faust and Raes, 2012). Recent studies have also highlighted other pitfalls in correlational analysis, particularly the accuracy of interactions identified even when the data reflects known modes of microbial interactions (Hirano and Takemoto, 2019; Carr et al., 2019).

Predictive and dynamic modeling of microbiomes based on first-order differential equations (e.g. with generalized Lotka-Volterra models or gLVMs) has found increasing usage and provided useful insights into microbial interactions and dynamics (Buffie et al., 2014; Bucci et al., 2016; Venturelli et al., 2018). Wider adoption of such techniques has been hampered by the need for large datasets (as the number of parameters grows quadratically with the number of species) and dense longitudinal sampling to adequately capture finegrained dynamics (Silverman et al., 2018). Theoretical assumptions such as the availability of data where all species are at equilibrium, and where absolute abundances are accurately known, make the determination of gLVM parameters from cross-sectional data solvable in principle (Xiao et al., 2017). In practice, metagenomic data provides relative abundances and scaling these accurately enough for gLVM parameter estimation can be challenging (Li et al., 2019). Furthermore, real-world datasets often contain a mixture of perturbed and unperturbed 
microbiomes where the equilibrium status is unknown, and where data may even come from multiple models (Arumugam et al., 2011).

Here we show that an expectation maximization algorithm which couples gLVM parameter inference with scaling factor estimation (BEEM - originally designed for longitudinal data [Li et al., 2019]) can be transformed to work with cross-sectional data from communities that are at or near equilibrium (BEEM-Static). In benchmarking comparisons with simulated communities against 10 other methods that infer microbial interactions from cross-sectional data, we noted that while all other methods only improved slightly over random predictions (AUC-ROC<0.63), BEEM-Static exhibited high accuracy similar to estimation using true scaling values (AUC-ROC>0.88). Similar observations were made with synthetic communities based on all-pair co-culture experiments, where BEEM-Static accurately recapitulated nearly all known interactions and their directionality. Based on statistical filters to identify non-model and/or non-equilibrium samples in real and simulated datasets, we show that BEEM-Static can be robust to up to $40 \%$ of data violating these assumptions. Applying BEEM-Static to a large public collection of human gut microbiome profiles $(n=4,617)$ identified multiple stable equilibria that appear to better reflect ecological enterotypes with distinct carrying capacities and interactions for key species (e.g. Prevotella copri) compared to prior clustering based definitions (Arumugam et al., 2011). BEEM-Static thus provides new opportunities for mining ecologically interpretable interactions and systems insights from the growing corpus of metagenomic data in the public domain.

\section{Methods}

\subsection{Estimating biomass and gLVM parameters with cross-sectional data}

The gLVM model is a set of differential equations describing the instantaneous growth rate of each species $\left(d x_{i}(t) / \mathrm{dt}\right)$ as a function of absolute cell densities $\left(x_{i}(t)\right)$ of the $p$ species in a community:

$$
\frac{d x_{i}(t)}{d t}=\mu_{i} x_{i}(t)+\sum_{j=1}^{p} \beta_{i j} x_{i}(t) x_{j}(t)
$$

where $\mu_{i}$ is the intrinsic growth rate of species $i$ and $\beta_{i j}$ are interaction terms that define the strength of the influence of species $j$ 's abundance on species $i$ 's growth. In general, estimating gLVM parameters $\left(\mu_{i}\right.$ and $\left.\beta_{i j}\right)$ requires longitudinal data to measure $d x_{i}(t) / d t$. However, at the non-trivial equilibrium $\left(d x_{i}(t) / d t=0\right.$ and $\left.x_{i}>0\right)$ : 


$$
\mu_{i}+\sum_{j=1}^{p} \beta_{i j} x_{j}=0
$$

where the time parameter $t$ now becomes implicit in the equation. Dividing both sides by $-\beta_{i i}$ and the biomass $m$, rearranging terms, and re-parameterizing the equation we get:

$$
\tilde{x}_{i}=\frac{a_{i}}{m}+\sum_{j=1, j \neq i}^{p} b_{i j} \tilde{x}_{j},
$$

where $a_{i}=-\mu_{i} / \beta_{i i}, b_{i j}=-\beta_{i j} / \beta_{i i}$ and $\tilde{x}_{i}$ is the relative abundance of species $i$ at equilibrium ( $a_{i}$ is also known as the carrying capacity of the species). This equation allows us to estimate gLVM parameters (through $a_{i}$ and $b_{i j}$ ) from cross-sectional data, assuming that samples are at equilibrium, and absolute abundances are known $\left(x_{i}=m \tilde{x}_{i}\right)$. To account for the fact that metagenomics provides relative abundances and biomass is typically not measured, BEEM-Static extends the following EM framework to jointly estimate model parameters and biomass (Li et al., 2019):

Estimating model parameters (E-step): BEEM-Static estimates model parameters $\left(a_{i}^{(T)}\right.$ and $\left.b_{i j}^{(T)}\right)$ for each species $i$ with sparse regression (implemented with the 'glmnet' package in $\mathrm{R}$ ) in iteration $T$ :

$$
\tilde{x}_{i} \sim \frac{1}{m^{(T-1)}} \cdot a_{i}^{(T)}+\sum_{j=1, j \neq i}^{p} b_{i j}^{(T)} \tilde{x}_{j} .
$$

Estimating biomass (M-step): for a sample, the equation for each species $i$ provides an estimate for the biomass, and BEEM-Static takes the median of estimates across species as a robust estimator for the biomass of the sample:

$$
m^{(T)}=\operatorname{median}\left(-\frac{a_{i}^{(T)}}{\sum_{j=1}^{p} b_{i j}^{(T)} \tilde{x}_{j}}\right) .
$$

Initialization and termination: BEEM-Static initializes biomass values based on normalization factors from cumulative sum scaling (CSS; Paulson et al., 2013), with a user defined scaling constant as the median of biomass values (kept constant through EM iterations). The EM process is then run until the maximum number of iterations specified (200 by default) or until convergence when the median of relative changes in biomass values is $<10^{-3}$. Confidence values (Z-scores) for the final interaction matrix (non-zero off-diagonal entries) were calculated for each species $i$ using forward stepwise regression (implemented in R package "selectiveInference"; version 1.2.5; Akaike information criterion as the stopping criterion). 


\subsection{Statistical filters to detect violations of modeling assumptions}

BEEM-Static uses the following filters to identify and remove samples that violate modeling assumptions and could thus impact model inference:

Equilibrium filter: to identify samples that may not be at equilibrium, BEEM-Static first predicts the abundances of species at equilibrium (based on the current model) for all species that are present:

$$
\widetilde{\boldsymbol{x}}^{*}=-\frac{1}{m^{(T-1)}}\left(\mathbf{B}^{-1} \boldsymbol{a}\right)
$$

where $\boldsymbol{B}$ is the matrix form of $\hat{b}_{i j}^{(T)}$ (= estimate for $\left.b_{i j}^{(T)}\right), \boldsymbol{a}$ is a column vector taking the value of $\hat{a}_{i}^{(T)}$ (= estimate for $a_{i}^{(T)}$ ) and $\widetilde{\boldsymbol{x}}^{*}$ is a vector of predicted relative abundances at equilibrium for each species ( $\tilde{x}_{i}{ }^{*}$ is set to 0 if species $i$ is not present). Samples with median relative deviation above a user defined threshold $\left(\epsilon_{1}\right)$ from these equilibrium values were then excluded as being potentially not at equilibrium $\left(\operatorname{median}\left(\left|\tilde{x}_{i}-\tilde{x}_{i}^{*}\right| / \tilde{x}_{i}\right)>\epsilon_{1}, \tilde{x}_{i} \neq 0\right.$ and $\epsilon_{1}=20 \%$ by default).

Model filter: to account for cases where some samples may come from an alternate gLVM, BEEM-Static calculates the median of squared errors for each sample $k$ with respect to the current model parameter estimates $\left(\hat{a}_{i}^{(T)}\right.$ and $\left.\hat{b}_{i j}^{(T)}\right)$ :

$$
e_{k}=\operatorname{median}_{\tilde{x}_{i} \neq 0}\left(\left(\tilde{x}_{i}-\left(\frac{1}{m^{(T-1)}} \cdot \hat{a}_{i}^{(T)}+\sum_{j=1, j \neq i}^{p} \hat{b}_{i j}^{(T)} \tilde{x}_{j}\right)\right)^{2}\right)
$$

This is done in the E-step for each iteration and samples with large median squared error, i.e. $\left(e_{k}-\operatorname{median}\left(e_{k}\right)\right) / \mathrm{IQR}\left(e_{k}\right)>\epsilon_{2}$ where IQR is the inter-quartile range and $\epsilon_{2}$ is a user defined parameter (default value of 3), are then removed for the next iteration's E-step.

\subsection{Selecting shrinkage parameters for sparse regression}

The shrinkage parameter $\lambda$ in the sparse regression penalizes the number of parameters to avoid overfitting and is selected based on five-fold cross-validation in each iteration (selecting the value one standard error away from the best $\lambda$; Friedman et al., 2010). In the Estep of iteration $T$, a crude selection of $\lambda_{c}^{(T)}$ is made in BEEM-Static from a large range from $10^{-10}$ to $10^{-1}$, and then refined with a fine-grained sequence from $\lambda_{c}^{(T)} / 10$ to $10 \lambda_{c}^{(T)}$.

\subsection{Generating simulated datasets}

Simulated gLVM data was generated based on previously described procedures (Berry and Widder, 2014; Bucci et al., 2016). Specifically, to parameterize distributions for generating 
model parameters, MDSINE (Bucci et al., 2016) was used to estimate the mean and standard deviations of growth rates and inter-/intra-species interaction parameters from the $C$. difficile infection experiment data provided with the software. Growth rates and intra-species interactions were sampled from normal distributions (forced to be positive and negative respectively to model logistic growth). The interaction network structure was generated by randomly adding edges from one species to another (with probability ranging from 0.1 to 0.5 ) and the magnitude of the interactions was sampled from a normal distribution (with 0 mean and standard deviation estimated from real data as noted above). Initial abundances of $p(30)$ species were sampled from a uniform distribution (from 0.001 to the mean carrying capacity $\mu_{i} / \beta_{i i}$ of all the $p$ species), with each species having a probability of $\pi$ to be absent from a sample ( $\pi$ was estimated as the average rate of absence for the top $p$ most prevalent species in all healthy gut microbiome profiles from the database curatedMetagenomicData [Pasolli et al., 2017]). A dataset with $n$ samples was generated by numerically integrating the gLVM with the same parameters until equilibrium, starting with $n$ different initial abundance profiles. The abundances of a random time point along the numerical integration ( $>20 \%$ away from the abundance at equilibrium for $>50 \%$ of the species) was selected as a sample not at equilibrium. Poisson noise was added to the abundance of each species to simulate experimental variability.

\subsection{Generating datasets based on growth curves and co-culture experiments}

Microbiome profiles for co-culture experiments were taken from a previous study (Venturelli et al., 2018) and the relative abundances were scaled using the corresponding biomass measurements (OD600). Six species pairs were randomly selected, and one of the three conditions were randomly picked for each pair in each sample: (1) only the first species was present, (2) only the second species was present and (3) both species were present. For the first two cases, a random timepoint (last 6 timepoints near the equilibrium) was taken from the growth curve (measured by OD600) of species present. For the last case, the scaled abundances of the two species near the steady state (randomly taken from the three replicates) were used. Abundances were re-scaled to relative abundances and the process repeated to generate a dataset with 500 samples. The interaction matrix reported in Venturelli et al (Venturelli et al., 2018) was treated as the ground truth ("M-PW1-PW2").

\subsection{Evaluation metrics}

We computed the median of relative errors to assess the accuracy of predicted parameters as: 


$$
\operatorname{median}\left(\frac{|\hat{\theta}-\theta|}{\max (|\hat{\theta}|,|\theta|)}\right)
$$

where $\hat{\theta}$ and $\theta$ are the estimated and true parameters $(\boldsymbol{a}, \boldsymbol{b}$ and $\boldsymbol{m})$ respectively. The area under the receiver operating characteristic curve (AUC-ROC) was computed for the interaction matrix. Z-scores were used to rank interactions (off-diagonal entries only) predicted by BEEM-Static. Sensitivity for predicting the signs of interaction was calculated as the fraction of interactions with correctly predicted signs in the true interaction matrix (non-zero off-diagonal entries only). Sign precision was computed as the fraction of interactions with correctly predicted signs.

\subsection{Benchmarking with correlation-based methods}

The following correlation-based methods for infering interactions from microbiome data were tested:

- Pearson and Spearman correlations calculated directly from relative abundances

- CCREPE (Faust et al., 2012; v1.2.0; 1000 iterations) corrected Pearson and Spearman correlation

- SparCC (Friedman and Alm, 2012; commit id: 9a1142c; default parameters)

- CCLasso (Fang et al., 2015; v1.0; default parameters)

- REBACCA (Ban et al., 2015; http://faculty.wcas.northwestern.edu/ hji403/REBACCA/REBACCA_main.R; nbootstrap=50, B=500, FWER=0.01)

- $\quad$ MInt (Biswas et al., 2014; v1.0.1; default parameters)

- SPIEC-EASI (Kurtz et al., 2015; v0.1.4; "mb" and "glasso"; lambda.min.ratio=0.01, nlambda $=20$, rep.num $=50$ )

- BAnOCC (Schwager et al., 2017; v1.0.1; default parameters)

- gCoda (Fang et al., 2017; commit id: 584bd07; default parameters)

The - $\log (\mathrm{p}$-value) or edge stability were used to rank CCREPE and SPIEC-EASI correlations, respectively, while the absolute values of correlation coefficients were used for the other methods. AUC-ROC was calculated from the lower triangle of the inferred correlation matrix (an inference was considered correct if there was an interaction between the corresponding species regardless of the interaction direction). Sensitivity and precision of signs were calculated as described above, excluding positive-negative interactions as they cannot be differentiated from positive-positive and negative-negative interactions using correlation analysis. 


\subsection{Analysis of gut microbiome data}

Healthy gut microbiome profiles from the database curatedMetagenomicData were preprocessed and used as the standard dataset for learning gLVMs by removing (1) replicate samples, (2) timepoints other than the first timepoint in longitudinal studies, (3) samples under antibiotic treatment and (4) samples from infants. In addition, we included two validation datasets to evaluate different aspects of the model learned by BEEM-Static: (1) all samples from Raymond et al (Raymond et al., 2016) to validate BEEM-Static's estimated growth and (2) samples from healthy infants (only the first timepoint for each subject) with ages below 12 months to evaluate BEEM-Static's biomass estimation. Both datasets were also used to validate BEEM-Static's ability to filter out samples violating model assumptions. To make the number of parameters tractable with the number of data points available, we only kept core species that were present (relative abundance $>0.1 \%$ ) in more than $30 \%$ of samples and subsequently removed samples where none of the core species were found, resulting in 42 core species and 4,617 samples overall. BEEM-Static was applied with the "model filter" $\left(\epsilon_{2}=0.9\right)$ to learn two models $(1,995$ and 1,145 samples for each model) in two iterations, in which samples violating the filter were removed. BEEM-Static was then rerun without the filter on samples assigned to each model separately to re-learn parameters.

\subsection{Estimating in situ growth using BEEM-Static and GRiD}

With BEEM-Static, in situ growth can be estimated as the deviation from equilibrium:

$$
\hat{a}_{i}+\widehat{m} \sum_{j=1}^{p} \hat{b}_{i j} \tilde{x}_{j}
$$

where $\hat{a}_{i}, \hat{m}$ and $\hat{b}_{i j}$ are estimated parameters. In addition, species replication rates for samples not under antibiotic treatment in Raymond et al (Raymond et al., 2016) were estimated with the high-throughput mode of GRiD (v1.2.0; default parameters) (Emiola and Oh, 2018). Gut microbiome associated genomes provided with GRiD were used as references and read reassignment using pathoscope2 (Hong et al., 2014) was enabled (parameter “-p") to resolve ambiguous mappings.

\subsection{Code availability}

BEEM-Static is available as an R package at https://github.com/CSB5/BEEM-static under the MIT license. 


\section{Results}

\subsection{Accurate inference of ecological interactions from cross-sectional metagenomic data}

To evaluate if ecological interactions can be inferred from cross-sectional data we first began by conducting extensive benchmarks on complex simulated communities where the truth is known (using gLVMs, number of species=30, equilibrium conditions; Methods). Consistent with prior reports (Hirano and Takemoto, 2019; Carr et al., 2019), we noted that all 10 stateof-the-art correlation-based methods provided performances that were only slightly better than random predictions in identifying true interactions, even while ignoring directionality (AUC-ROC $<0.63$; Fig. 1A, Sup. Fig. 1). The ability to correct for compositionality of metagenomic data (CCREPE, SparCC, CCLasso, REBACCA), or reduce false positives from transitive correlations (MInt, SPIEC-EASI, BAnOCC and gCoda), did not notably change performance compared to naïve correlation calculations (Pearson or Spearman) when inferring ecological interactions. In contrast, BEEM-Static was able to infer the interactions with notably higher sensitivity and specificity than all correlation-based methods (AUCROC=0.88; Fig. 1A, Sup. Fig. 1). Although BEEM-Static only used noisy relative abundances for gLVM inference, its performance matched that of a positive control that assumes noise-free biomass values to scale relative abundances to absolute abundances (Fig. 1A, Sup. Fig. 1, BEEM-Static vs. true biomass), an assumption that is unlikely to be met in realistic settings (Li et al., 2019).

In addition to knowing that two species are interacting, a key aspect of ecological interactions is the directionality/sign of interactions, with correlation-based methods either exhibiting low sensitivity (REBACCA, SPIEC-EASI) or low precision (Pearson, Spearman, CCREPE, SparCC, CCLasso, MInt, BAnOCC, gCoda) despite the exclusion of predatory interactions (positive-negative) when calculating their performance (Fig. 1B; Methods). BEEM-Static addresses this issue by providing high sensitivity (>80\%) and precision (nearly $100 \%$ ) using cross-sectional metagenomic data (Fig. 1B). These observations were recapitulated in a wide range of simulated datasets with varying network structure and edge sparsity, highlighting BEEM-Static's robustness (Sup. Fig. 2, Sup. Fig. 3). Furthermore, BEEM-Static provides estimates for biomass values that were found to be consistently accurate (relative error $<10 \%$ ) and can be used to provide meaningful biological insights ( $\mathrm{Li}$ et al., 2019).

We extended the evaluations to experimental data, using all-pair co-culture and isolate growth curves for 12 species (Venturelli et al., 2018) to create synthetic communities where 
the interaction network is known, albeit sparse (Fig. 1C; Ground truth). Not surprisingly, recapitulating the structure of such a simple interaction network was not difficult for most correlation-based methods, with BAnOCC having the best performance overall (AUCROC $=0.9$, Sup. Fig. 4). However, determining directionality of interactions was still a challenge, despite the simplicity of the network. For example, in the case of BAnOCC the commensalistic interaction between DP and FP was captured as a negative correlation, while the predatory interaction between BH and ER was captured as a positive correlation (Fig. 1C; BAnOCC). BEEM-Static, on the other hand, was able to capture all interactions and their directionality correctly, except for one false positive (FP to DP) and one false negative (BT to EL) involving interactions with weak strength (Fig. 1C; BEEM-Static). BEEM-Static's utility in such datasets was consistently observed in comparison to correlation-based methods with AUC-ROC close to 1 (Sup. Fig. 4).

\subsection{Statistical filters in BEEM-Static provide robustness to violations in modeling assumptions}

While the simulations in the previous section account for experimental errors, they assume that all samples come from equilibrium states, an unlikely situation for most real datasets. Relaxing this assumption, we noted that with as little as $5 \%$ non-equilibrium samples, AUCROC decreased by $>10 \%$, and with $15 \%$ non-equilibrium samples AUC-ROC performance degraded to match that of correlation-based methods (Naïve algorithm, Fig. 2A, Sup. Fig. 5A; Methods). Incorporating a statistical filter in BEEM-Static that compares estimated species relative abundances at equilibrium with observed abundances (equilibrium filter; Methods) helped identify samples that were not at equilibrium with high sensitivity and specificity (Sup. Fig. 5A). This in turn allowed BEEM-Static to be robust to having nearly half of the samples (45\%) in the dataset being at a non-equilibrium state (performance reduction $<5 \%$; Fig. 2A, Sup. Fig. 5A, BEEM-Static).

We next investigated the impact of relaxing the "universal model" assumption i.e. that all samples have the same ecological conditions and model parameters. This assumption may not hold true in many real-world settings (e.g. gut microbiome samples from different enterotypes [Arumugam et al., 2011]), and as expected relaxing it had a strong impact (30\% reduction in AUC-ROC) even with slight deviations (5\%, Naïve algorithm; Fig. 2B, Sup. Fig. 5B; Methods). In addition, AUC-ROC performance continued to decrease beyond $60 \%$ even after nearly half the samples (40-45\%) were derived from a different model. To address this, BEEM-Static implements a filter that identifies samples that have poor goodness-of-fit 
to the current model (model filter; Methods) and excludes them in subsequent iterations of model inference. This approach was found to provide robustness to up to $\sim 40 \%$ of samples from a different model (performance reduction $<20 \%$, BEEM-Static; Fig. 2B, Sup. Fig. 5B).

Finally, we employed real microbiome datasets to test BEEM-Static's robustness where a subset of samples is known to violate modeling assumptions i.e. some subjects undergoing oral antibiotic treatment or samples from newborn infants, where the majority of samples are from adults who are not undergoing antibiotic treatment. BEEM-Static was able to identify such samples with high sensitivity (>80\%) using its model filter (Fig. 2C, D), and the filtered samples were significantly enriched for those from antibiotic-treated adults and infants (Fisher's Exact test p-value $<10^{-22}$ ).

\subsection{BEEM-Static analysis of human gut microbiomes identifies distinct ecological configurations}

To further assess BEEM-Static's utility we evaluated the concordance of parameters learnt during the training process with orthogonal information for a large human gut microbiome dataset ( $\mathrm{N}=4,617$; Methods). In particular, we noted that biomass estimates from BEEMStatic were significantly higher for adults versus newborn infants $(\sim 2 \times$; Wilcoxon test $\mathrm{p}$ value $<10^{-15}$; Fig. 3A), consistent with our understanding of a maturing gut microbiome (Tsuji et al., 2018; Hopkins et al., 2005). Additionally, we used deviations from equilibrium $\left(d x_{i}(t) / d t=0\right)$ to estimate instantaneous growth (population increase or decrease) of each species in each sample (Methods), and assessed concordance with an in silico approach to estimate DNA replication rates (Emiola and Oh, 2018). Despite the fact that growth rates are also impacted by death rates, we observed that species predicted to grow based on BEEMStatic analysis were also found to have significantly higher DNA replication rates (GRiD values; Wilcoxon test $\mathrm{p}$-value $=3 \times 10^{-4} ; \mathbf{F i g}$. 3B).

Using an iterative approach to train a new model on samples excluded from the first model, we observed that BEEM-Static classifies a majority of the samples to two models (1995 for model 1 and 1145 for model 2; Sup. Table 1). Visualizing the expected microbial composition for each sample at equilibrium, we noted three distinct clusters in a principle component analysis plot (Fig. 3C), where samples in clusters 1 and 2 largely correspond to profiles from models 1 and 2, respectively. Analysis of carrying capacities highlighted that the clusters were defined by Prevotella copri, which has higher carrying capacity in cluster 1 vs 2 and is absent in samples from cluster 3 (Fig. 3D). Several other species were also found to have divergent carrying capacities in the two models, including Bifidobacterium 
adolescentis, Collinsella aerofaciens and Coprococcus comes (enriched in model 2), some of which have variations associated with fiber intake (Adamberg et al., 2020; Wu et al., 2011). Overall the ecological models identified here have distinct sets of interactions (Sup. Table 1) and do not appear to match earlier definitions of enterotypes (Arumugam et al., 2011) based on principle coordinate analysis and clustering (Sup. Fig. 6).

\section{Discussion}

As microbiome research increasingly moves from descriptive studies to those that seek to provide a mechanistic understanding of microbial communities, the ability to infer microbial interactions from metagenomic data is an important capability. In particular, the directionality and sign of interactions provide biologically interpretable information that is missed by correlation-based approaches. BEEM-Static provides an alternative avenue to infer this, with the caveat that it assumes a specific model (generalized Lotka-Volterra) for community dynamics. In addition, as we show here, other values obtained from BEEM-Static models can have utility, including the strength of interactions, biomass estimates, deviation from equilibrium, and fit to model.

In addition to accounting for relative abundance estimates from metagenomic data, the statistical filters employed by BEEM-Static make it robust to some of the violations in model assumptions that can be expected in real datasets. These features make BEEM-Static widely applicable, and also extends the use of ecological models with metagenomic data. For instance, our analysis of large public metagenomic datasets provides an alternate perspective to the discussion on microbial enterotypes (Arumugam et al., 2011) and universality of microbiome dynamics (Bashan et al., 2016). The ecological types observed here are characterized by distinct carrying capacities that might be a function of the environment (e.g. host factors or diet). Fiber rich diets are known to have a strong impact on the gut microbiome (Wu et al., 2011) and have been linked to some of the species with differential carrying capacities in our models (Adamberg et al., 2020). We anticipate that the incorporation of such environmental factors into future models would be an exciting avenue to study their influence on microbial community structure in vivo. Finally, hybrid methods that learn models from both longitudinal and cross-sectional data represent another promising direction to explore for studying general and individual specific microbiome dynamics (Li et al., 2019). 


\section{Acknowledgements}

We thank Dr. Swaine Chen, Dr. Rohan Williams and Dr. Jonathan Teo for their constructive comments and feedback on this work.

\section{Funding}

This work has been supported by A*STAR and a BMRC IAF-PP grant: H18/01/a0/016.

Conflict of Interest: none declared. 


\section{References}

Adamberg,K. et al. (2020) The composition of faecal microbiota is related to the amount and variety of dietary fibres. Int. J. Food Sci. Nutr., 71, 845-855.

Angulo,M.T. et al. (2019) A theoretical framework for controlling complex microbial communities. Nat. Commun., 10, 1045.

Arumugam,M. et al. (2011) Enterotypes of the human gut microbiome. Nature, 473, 174-80.

Ban,Y. et al. (2015) Investigating microbial co-occurrence patterns based on metagenomic compositional data. Bioinformatics, 31, btv364-.

Bashan,A. et al. (2016) Universality of human microbial dynamics. Nature, 534, 259-262.

Berry,D. and Widder,S. (2014) Deciphering microbial interactions and detecting keystone species with co-occurrence networks. Front. Microbiol., 5, 219.

Biswas,S. et al. (2014) Learning microbial interaction networks from metagenomic count data. J. Comput. Biol., 23, 526-535.

Bucci,V. et al. (2016) MDSINE: Microbial Dynamical Systems INference Engine for microbiome time-series analyses. Genome Biol., 17, 121.

Buffie,C.G. et al. (2014) Precision microbiome reconstitution restores bile acid mediated resistance to Clostridium difficile. Nature, 517, 205-208.

Carr,A. et al. (2019) Use and abuse of correlation analyses in microbial ecology. ISME J., 1.

Chng,K.R. et al. (2020) Metagenome-wide association analysis identifies microbial determinants of post-antibiotic ecological recovery in the gut. Nat. Ecol. Evol., 1-12.

Chng,K.R. et al. (2016) Whole metagenome profiling reveals skin microbiome-dependent susceptibility to atopic dermatitis flare. Nat. Microbiol., 1, 16106.

Clemente,J.C. et al. (2012) The impact of the gut microbiota on human health: An integrative view. Cell, 148, 1258-1270.

Cordero,O.X. and Datta,M.S. (2016) Microbial interactions and community assembly at microscales. Curr. Opin. Microbiol., 31, 227-234.

Emiola,A. and Oh,J. (2018) High throughput in situ metagenomic measurement of bacterial replication at ultra-low sequencing coverage. Nat. Commun., 9, 4956.

Fang,H. et al. (2015) CCLasso: Correlation Inference for Compositional Data through Lasso. Bioinformatics, 31, btv349-.

Fang,H. et al. (2017) gCoda: Conditional Dependence Network Inference for Compositional Data. J. Comput. Biol., 24, 699-708.

Faust,K. et al. (2012) Microbial co-occurrence relationships in the human microbiome. PLoS 
Comput. Biol., 8, e1002606.

Faust,K. and Raes,J. (2012) Microbial interactions: from networks to models. Nat. Rev.

Microbiol., 10, 538-50.

De Filippis,F. et al. (2018) Recent Past, Present, and Future of the Food Microbiome. Annu.

Rev. Food Sci. Technol., 9, 589-608.

Fraune,S. et al. (2015) Bacteria-bacteria interactions within the microbiota of the ancestral metazoan Hydra contribute to fungal resistance. ISME J., 9, 1543-1556.

Friedman,J. et al. (2010) Regularization Paths for Generalized Linear Models via Coordinate Descent. J. Stat. Softw., 33, 1-22.

Friedman,J. and Alm,E.J. (2012) Inferring Correlation Networks from Genomic Survey Data. PLoS Comput. Biol., 8, e1002687.

Gould,A.L. et al. (2018) Microbiome interactions shape host fitness. Proc. Natl. Acad. Sci. U. S. A., 115, E11951-E11960.

Hirano,H. and Takemoto,K. (2019) Difficulty in inferring microbial community structure based on co-occurrence network approaches. BMC Bioinformatics, 20, 329.

Hong,C. et al. (2014) PathoScope 2.0: a complete computational framework for strain identification in environmental or clinical sequencing samples. Microbiome, 2, 33.

Hopkins,M.J. et al. (2005) Characterisation of intestinal bacteria in infant stools using realtime PCR and northern hybridisation analyses. FEMS Microbiol. Ecol., 54, 77-85.

Kurtz,Z.D. et al. (2015) Sparse and Compositionally Robust Inference of Microbial

Ecological Networks. PLoS Comput. Biol., 11, e1004226.

Li,C. et al. (2019) An expectation-maximization algorithm enables accurate ecological modeling using longitudinal microbiome sequencing data. Microbiome, 7, 118.

Li,C. et al. (2016) Predicting Microbial Interactions through Computational Approaches. Methods.

Mohanram,S. and Kumar,P. (2019) Rhizosphere microbiome: revisiting the synergy of plantmicrobe interactions. Ann. Microbiol., 69, 307-320.

Pasolli,E. et al. (2017) Accessible, curated metagenomic data through ExperimentHub. Nat. Methods, 14, 1023-1024.

Paulson,J.N. et al. (2013) Differential abundance analysis for microbial marker-gene surveys. Nat. Methods, 10, 1200-1202.

Raymond,F. et al. (2016) The initial state of the human gut microbiome determines its reshaping by antibiotics. ISME J., 10, 707-720.

Röttjers,L. and Faust,K. (2018) From hairballs to hypotheses-biological insights from 
microbial networks. FEMS Microbiol. Rev., 42, 761-780.

Schwager,E. et al. (2017) A Bayesian method for detecting pairwise associations in compositional data. PLOS Comput. Biol., 13, e1005852.

Silverman,J.D. et al. (2018) Dynamic linear models guide design and analysis of microbiota studies within artificial human guts. Microbiome, 6, 202.

Stein,R.R. et al. (2018) Computer-guided design of optimal microbial consortia for immune system modulation. Elife, 7.

Tsuji,H. et al. (2018) Counting the Countless: Bacterial Quantification by Targeting rRNA Molecules to Explore the Human Gut Microbiota in Health and Disease. Front. Microbiol., 9, 1417.

Venturelli,O.S. et al. (2018) Deciphering microbial interactions in synthetic human gut microbiome communities. Mol. Syst. Biol., 14, e8157.

Wu,G.D. et al. (2011) Linking long-term dietary patterns with gut microbial enterotypes. Science (80-. )., 334, 105-108.

Xiao, Y. et al. (2020) An ecological framework to understand the efficacy of fecal microbiota transplantation. Nat. Commun., 11, 1-17.

Xiao,Y. et al. (2017) Mapping the ecological networks of microbial communities. Nat.

Commun., 8, 2042. 


\section{Figure Legends}

Fig. 1. Benchmarking performance for network structure and edge directionality. Note that CCREPE has two versions with Pearson and Spearman correlations (CCREPE.P and CCREPE.S), while SPIEC-EASI using "mb" and "glasso" algorithms is represented as SE.mb and SE.glasso, respectively. (A) ROC curves for different correlation based methods, the regression method using true biomass values and BEEM-Static for one simulated dataset with 30 species and 500 samples. (B) Boxplots showing precision and recall for directionality/sign of interactions for 30 different simulated communities with 30 species and 500 samples each. (C) True interaction network for a synthetic community based on all-pair co-culture data (Ground truth), inferred correlation network by BAnOCC and inferred interaction network by BEEM-Static.

Fig. 2. BEEM-Static robustly filters samples violating modeling assumption in simulated and real datasets. (A-B) Performance reduction for BEEM-Static (with filters) and the naïve algorithm (without filtering of samples) as the percentage of samples at (A) equilibrium or (B) generated from the main model, decreases. Reduction is measured relative to BEEM-Static with no filters and all data from the model and at equilibrium. Points denote the means while error bars denote the standard deviation across 30 simulations each. (C-D) Principle coordinates plots (Bray-Curtis dissimilarity) representing gut microbiome taxonomic profiles from 4,617 samples. Points represent samples from individuals taking antibiotics (C) or from newborn infants (D), while crosses represent samples from adults who are not undergoing antibiotic treatment. Points that were filtered by BEEM-Static are colored blue and red otherwise.

Fig. 3. Analysis of human gut microbiomes with BEEM-Static. (A) Violin plots showing the significant difference in BEEM-Static biomass estimates for adults and newborn infants. (B) Boxplots showing differences in DNA replication rates (GRiD estimates) for species predicted to decrease and increase in population size, respectively, by BEEM-Static. Each point represents one species in a sample. (C) Scatter plot showing the first two components from a principle component analysis of equilibrium abundances for samples (as predicted by BEEM-Static). (D) Predicted carrying capacities (square root transformed) of species in the two models. Species with divergent carrying capacities (ratio is $>2$ standard deviations from 1) in the two models are marked with stars. 


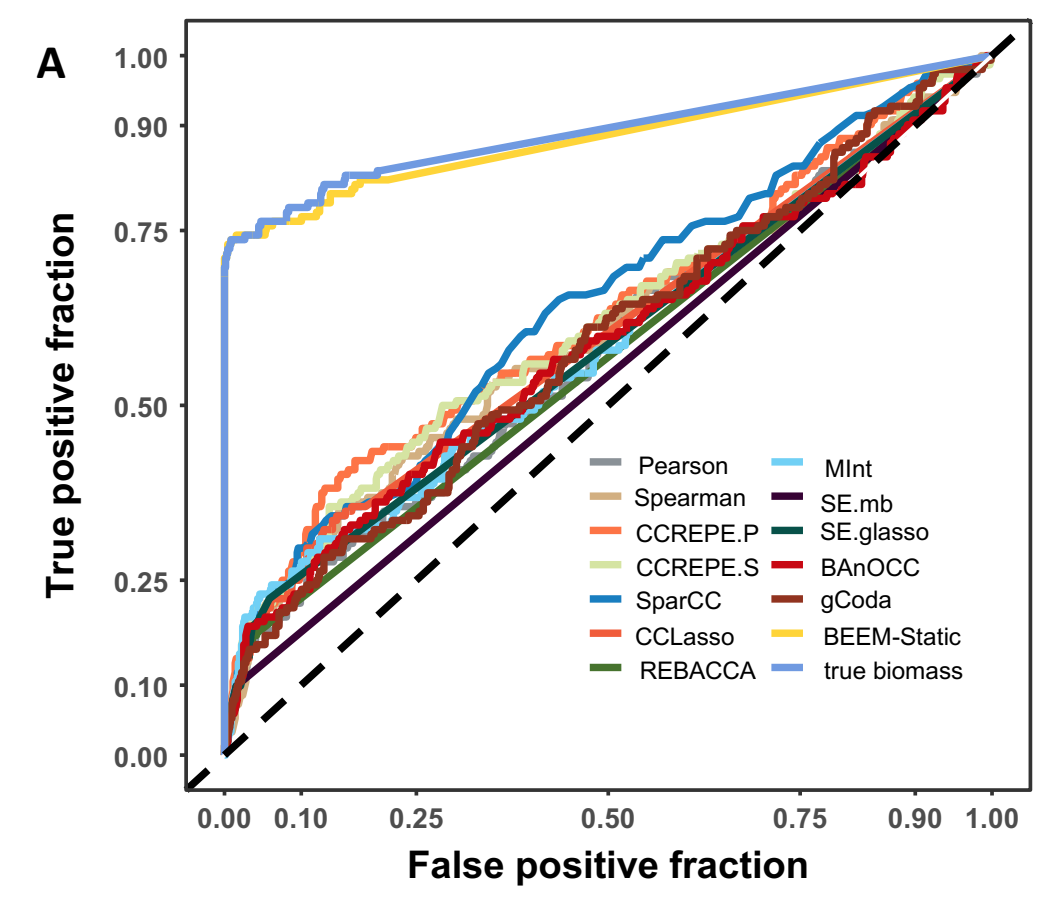

B

\section{Sign sensitivity}

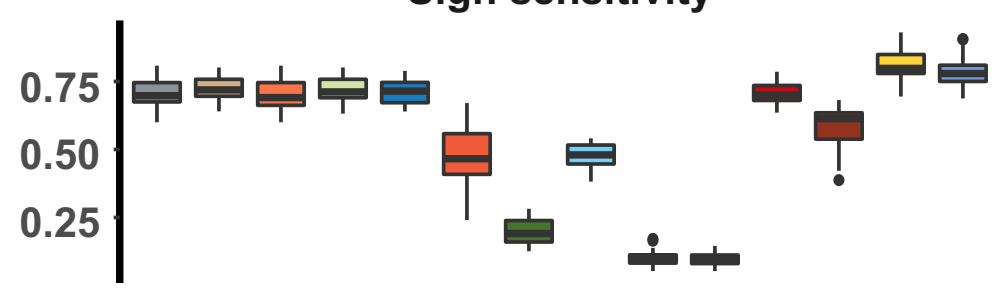

\section{Sign precision}

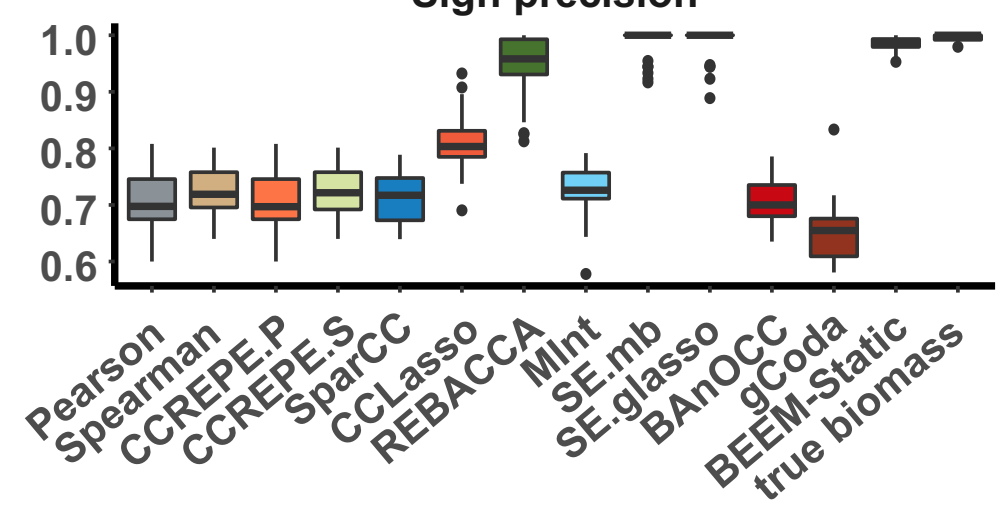

C

Ground truth
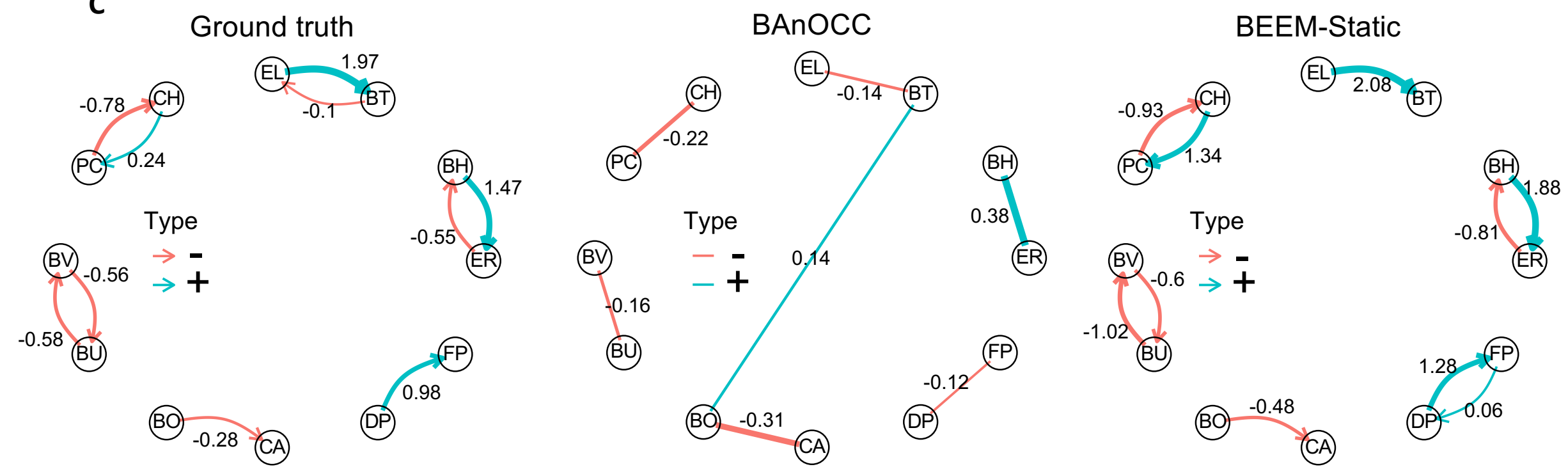
A
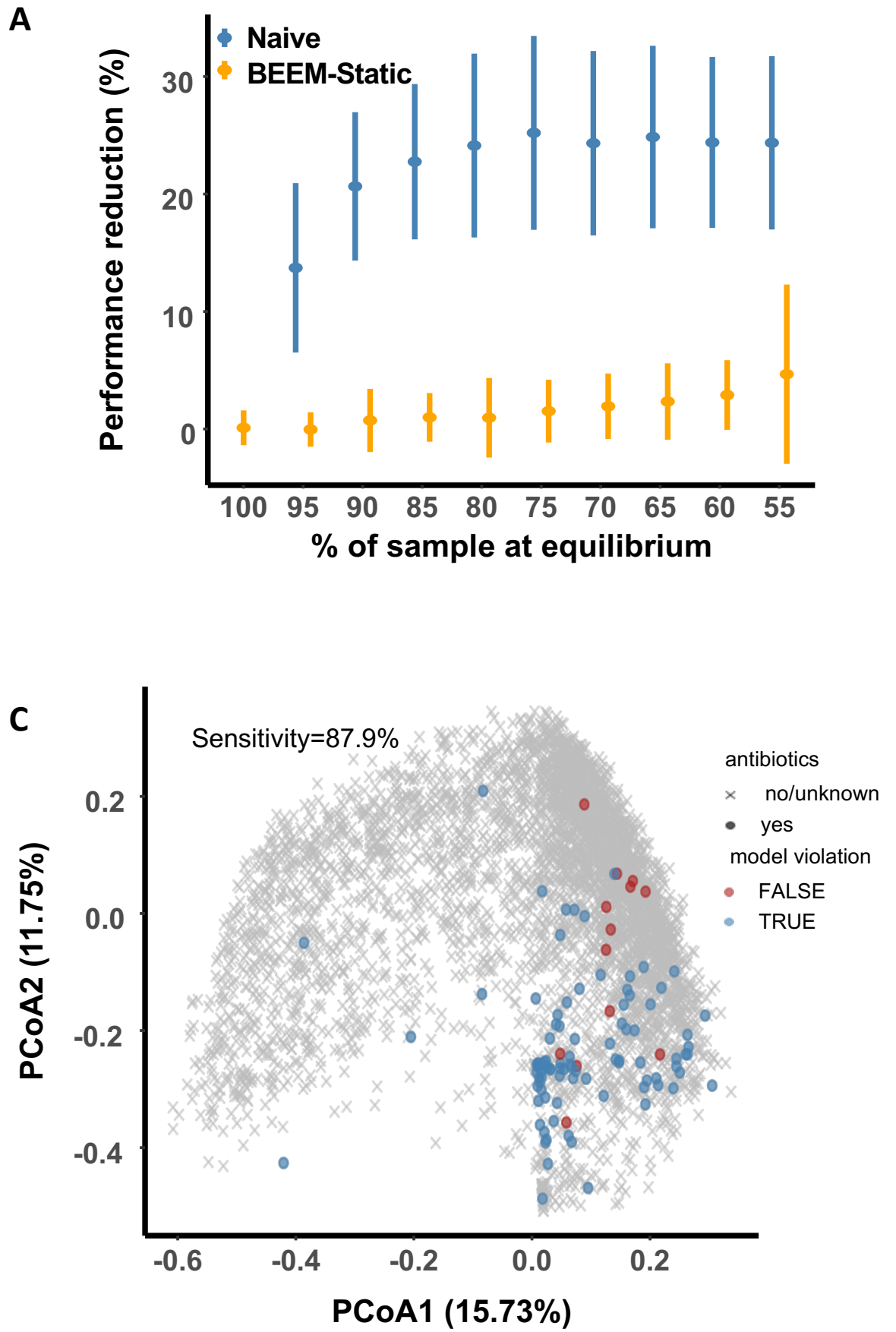

B
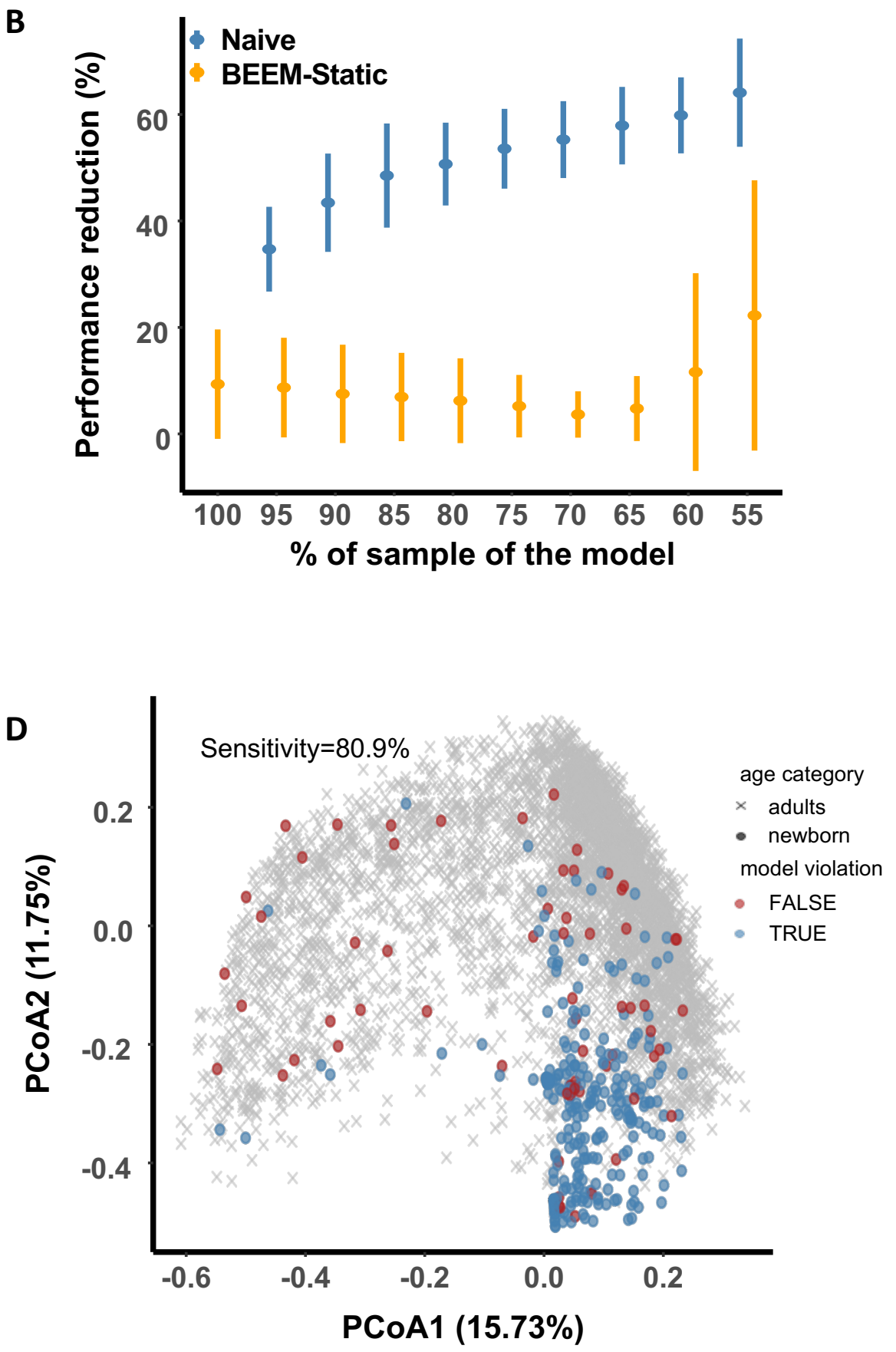

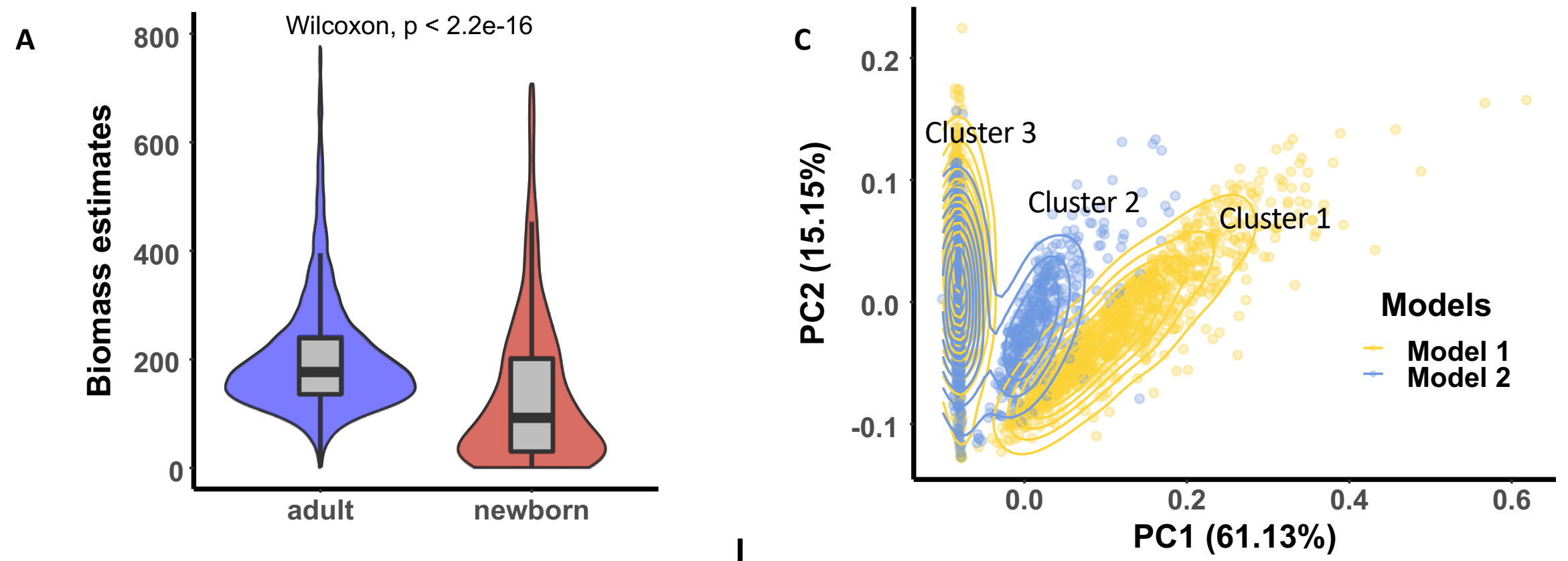

B

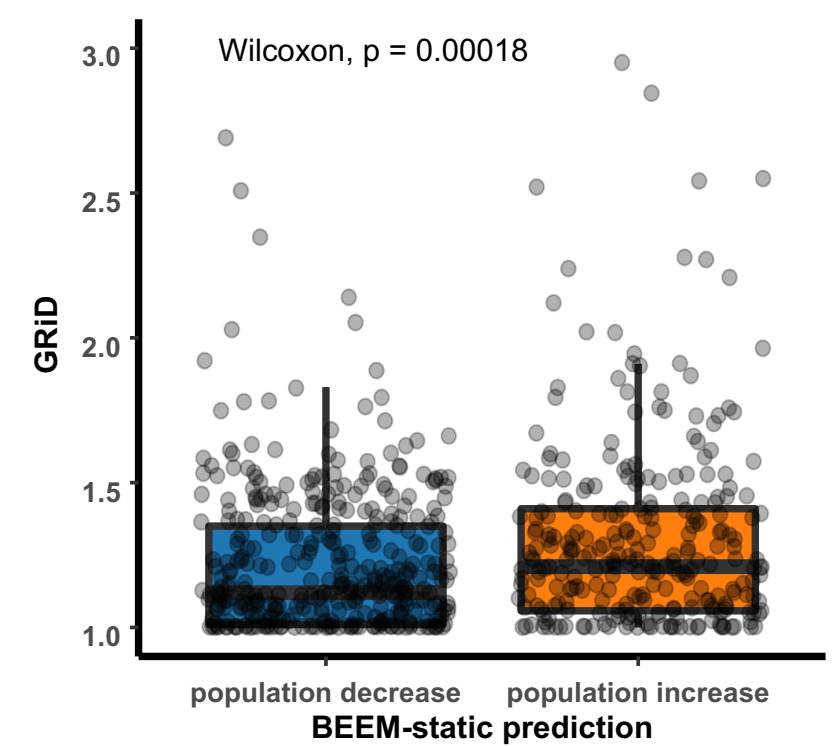

D

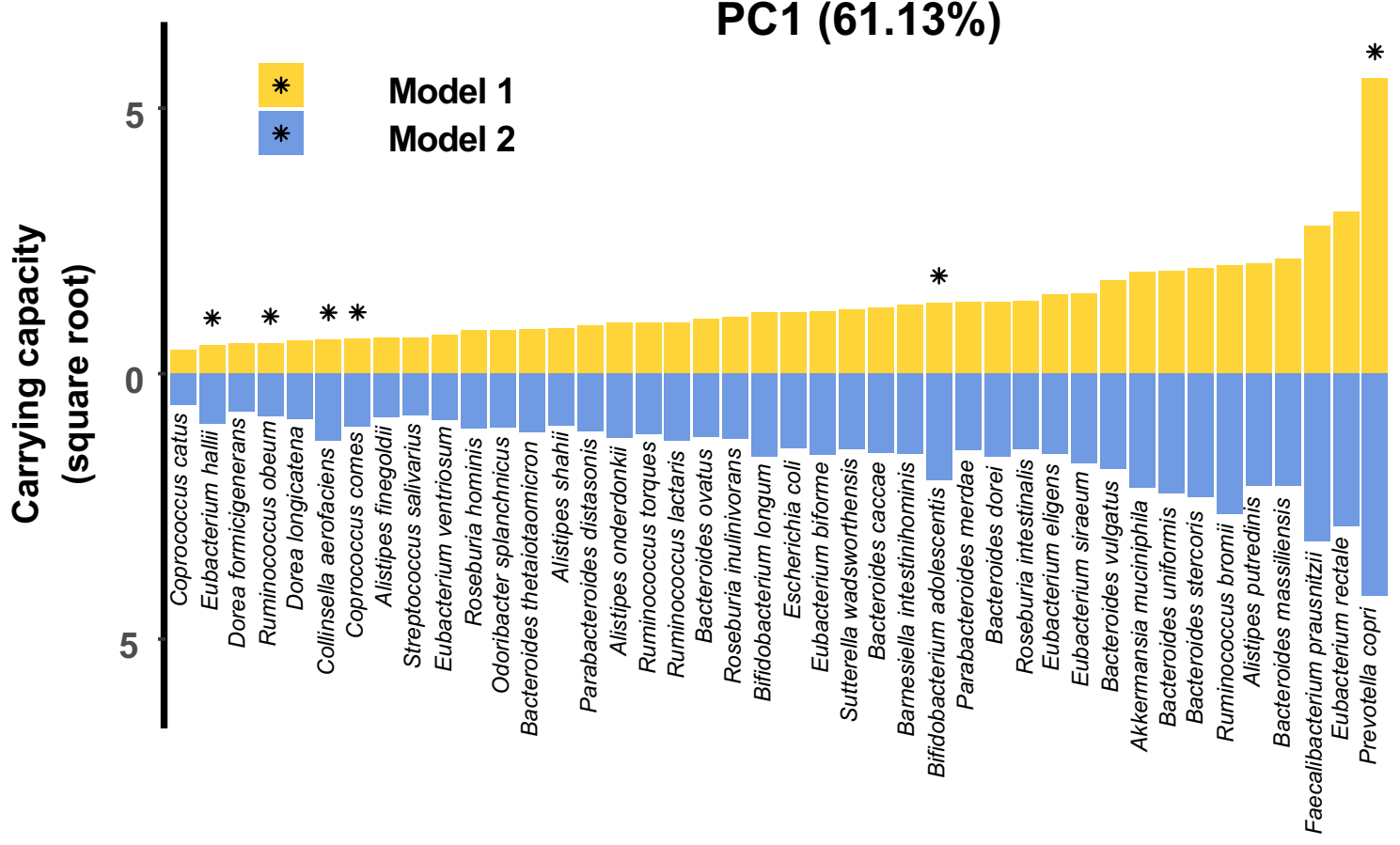

species 